\title{
Regulation of S1P receptors and sphingosine kinases expression in acute pulmonary endothelial cell injury
}

\author{
Huiying Liu ${ }^{1}$, Zili Zhang ${ }^{1}$, Puyuan Li ${ }^{1}$, Xin Yuan ${ }^{1}$, Jing Zheng ${ }^{1}$, Jinwen Liu ${ }^{2}$, Changqing Bai ${ }^{\text {Corresp.., }}{ }^{1}$, \\ Wenkai Niu Corresp. 1 \\ 1 Department of Respiratory and critical care diseases, 307th Hospital of PLA, Beijing, the People's Republic of China \\ 2 Beijing Oriental Yamei Gene Science \& Technology Institute, Beijing, the People's Republic of China \\ Corresponding Authors: Changqing Bai, Wenkai Niu \\ Email address: mlp1604@sina.com, niuwk88@sina.com
}

Background. Acute lung injury and acute respiratory distress syndrome (ALI /ARDS) is a severe clinical syndrome with mortality rate as high as $30-40 \%$. There is no treatment yet to improve pulmonary endothelial barrier function in patients with severe pulmonary edema. Developing therapies to protect endothelial barrier integrity and stabilizing gas exchange is getting more and more attention. Sphingosine-1-phosphate (S1P) is able to enhance the resistance of endothelial cell barrier. S1P at physiological concentrations plays an important role in maintaining endothelial barrier function. Proliferation, regeneration and anti-inflammatory activity that mesenchymal stem cells (MSCs) exhibit make it possible to regulate the homeostatic control of S1P.

Methods. By building a pulmonary endothelial cell model of acute injury, we investigated the regulation of S1P receptors and sphingosine kinases expression by MSCS during the treatment of acute lung injury using RT-PCR, and investigated the HPAECS Micro-electronics impedance using Real Time Cellular Analysis.

Results. It was found that the down-regulation of TNF- $\alpha$ expression was more significant when MSC was used in combination with S1P. The combination effection mainly worked on S1PR2, S1PR3 and SphK2. The results show that when MSCs were used in combination with S1P, the selectivity of S1P receptors was increased and the homeostatic control of S1P concentration was improved through regulation of expression of S1P metabolic enzymes. Discussions. The study found that as a potential treatment, MSCs could work on multiple S1P related genes, simultaneously. When it was used in combination with S1P, the expression regulation result of related genes was not simply the superposition of each other, but more significant outcome was obtained. This study establishes the experimental basis for, further exploring the efficacy of improving endothelial barrier function in acute lung injury, using MSCs in combination with S1P and their possible synergistic mechanism. 
3

4 Huiying Liu ${ }^{1}$, Zili Zhang ${ }^{1}$, Puyuan $\mathrm{Li}^{1}$, Xin Yuan ${ }^{1}$, Jing Zheng ${ }^{1}$, Jinwen Liu², Changqing Bai ${ }^{1}$, 5 Wenkai Niu ${ }^{1}$

61 Department of Respiratory and critical care diseases, 307th Hospital of PLA, Beijing, the

7 People's Republic of China

82 Beijing Oriental Yamei Gene Science \& Technology Institute, Beijing, the People's Republic of 9 China

\section{Regulation of S1P receptors and sphingosine kinases expression in acute pulmonary endothelial cell injury}

Corresponding Author:

Wenkai Niu ${ }^{1}$

No. 8 Dongdajie Street, Fengtai District, Beijing, 100071, P. R. China

Email address: niuwk88@sina.com

Changqing Bai ${ }^{1}$

No. 8 Dongdajie Street, Fengtai District, Beijing, 100071, P. R. China

Email address: mlp1604@sina.com

\section{Abstract:}

Background. Acute lung injury and acute respiratory distress syndrome (ALI /ARDS) is a severe clinical syndrome with mortality rate as high as $30-40 \%$. There is no treatment yet to improve pulmonary endothelial barrier function in patients with severe pulmonary edema. Developing therapies to protect endothelial barrier integrity and stabilizing gas exchange is getting more and more attention. Sphingosine-1-phosphate (S1P) is able to enhance the resistance of endothelial cell barrier. S1P at physiological concentrations plays an important role in maintaining endothelial barrier function. Proliferation, regeneration and anti-inflammatory activity that mesenchymal stem cells (MSCs) exhibit make it possible to regulate the homeostatic control of S1P.

Methods. By building a pulmonary endothelial cell model of acute injury, we investigated the regulation of S1P receptors and sphingosine kinases expression by MSCs during the treatment of acute lung injury using RT-PCR, and investigated the HPAECs Micro-electronics impedance using Real Time Cellular Analysis. 
31 Results. It was found that the down-regulation of TNF- $\alpha$ expression was more significant when

32 MSC was used in combination with S1P. The combination effect mainly worked on S1PR2, S1PR3

33 and SphK2. The results show that when MSCs were used in combination with S1P, the selectivity 34 of S1P receptors was increased and the homeostatic control of S1P concentration was improved 35 through regulation of expression of S1P metabolic enzymes.

36 Discussions. The study found that as a potential treatment, MSCs could work on multiple S1P 37 related genes, simultaneously. When it was used in combination with S1P, the expression 38 regulation result of related genes was not simply the superposition of each other, but more 39 significant outcome was obtained. This study establishes the experimental basis for, further 40 exploring the efficacy of improving endothelial barrier function in acute lung injury, using MSCs 41 in combination with S1P and their possible synergistic mechanism. 


\section{Introduction}

Acute lung injury and acute respiratory distress syndrome (ALI/ARDS) was first recognized as a clinical syndrome in the 1960s. It manifests as severe and acute respiratory failure with hypoxemia and lung infiltration and is often caused by pneumonia, sepsis and major trauma. The mortality rate of ALI/ARDS is as high as 30-40\% (Rubenfeld et al. 2005). In the large number of drugs evaluated by clinical trials, none has proven to be effective or could be recommended as the standard treatment of ALI/ARDS(Cepkova \& Matthay 2006). Supportive therapy is currently the major treatment, including protective ventilation and conservative medication (Brower et al. 2000; Wiedemann et al. 2006). Mechanical ventilation is a necessary and life-saving method, but it may delay the inflammatory response and ultimately results in pulmonary endothelial barrier dysfunction. Endothelial barrier dysfunction can lead to increased permeability, extravasation of fluid rich in proteins and pulmonary edema, which are all common symptoms of ALI/ARDS (Kumar et al. 2008). There is currently no treatment to improve pulmonary endothelial barrier function in severe pulmonary edema patients (Müller-Redetzky et al. 2014). Developing a therapy to protect endothelial barrier integrity and stabilize gas exchange is getting a lot of attention.

Sphingosine-1-phosphate (S1P) is a ubiquitous sphingomyelin and is an important regulator of vascular endothelial cell permeability and fluid balance in vivo. It is mainly present in plasma and tissues and is able to enhance the resistance of endothelial barrier. Endothelial barrier enhancement mediated by S1P is completed by activating the Gi and Racl signaling pathway through S1P receptor (Sun et al. 2012). Studies have demonstrated that S1P plays an important role in allergic reactions in the respiratory system. S1P promotes the adhesion of endothelial cells, which is the key in maintaining endothelial barrier and avoiding increased permeability leading to pulmonary edema (Lee et al. 1999). FTY720, structure analogue of S1P, was approved by FDA in the treatment of multiple sclerosis in 2010. FTY720 slightly differs from S1P in receptor binding activity, but they both have strong side effects at high doses (Natarajan et al. 2013). This indicates that in the treatment of lung disease, selectivity of S1P receptors and homeostatic control of S1P concentration is important. 
69

70

Several studies have demonstrated that $\mathrm{S} 1 \mathrm{P}$, at physiological concentration, has an important role in maintaining endothelial barrier function. For S1P and its structure analogues, their limitations in therapeutic function, confirm the conception of sphingolipid homeostasis. The fate of the cells is determined by the homeostasis of S1P concentration (Cuvillier et al. 1996). As an endogenous bioactive molecule, $\mathrm{S} 1 \mathrm{P}$ is an important regulator of vascular endothelial cell permeability and fluid balance. Its anabolic and key molecules on signaling pathways are still potential targets and focus of research in the treatment of ALI/ARDS.

Recent clinical studies have found that it is unlikely for any single drug to reverse the severe pathological injury caused by acute lung injury. Therefore, in the therapy of ALI/ARDS, there has been attention focused on cell therapy, especially mesenchymal stem cell therapy.

Mesenchymal stem cells (MSCs) are multi-functional cells with the ability of self-renewal. They were first discovered in bone marrow and can differentiate into bone, cartilage, fat, muscle and other tissues. At present, MSCs have demonstrated great efficacy in the treatment of cirrhosis (Zheng et al. 2012), systemic lupus erythematosus(Wang et al. 2013) and other diseases. Therapeutic effects have also been obtained in preclinical studies of Crohn's disease (Valcz et al. 2011), traumatic brain injury (Harting et al. 2009), sepsis (Nemeth et al. 2008), acute renal failure(Bruno et al. 2009) and other diseases. In recent years, several studies have reported, using a number of animal models of lung disease that allograft MSC therapy can reduce lung injury? (Chang et al. 2009; Moodley et al. 2009; Ortiz et al. 2003)

Most recent studies involving MSCs in the treatment of ALI/ARDS are focused on the evaluation of efficacy, with no further discussion of the immunomodulatory impact of MSCs on pulmonary tissue cells, especially the endothelial cell barrier. The regulation of S1P metabolism by MSCs is lacking in many systematic and comprehensive studies too. Homeostatic control of $\mathrm{S} 1 \mathrm{P}$ in vivo is a prerequisite to improving endothelial barrier and treating acute lung injury. The anabolic process of S1P is regulated by many cytokines. The outcomes of many diseases when MSCs were used demonstrate that it can secrete various growth and inflammatory factors, playing a role in immune regulation. Besides, according to some studies, S1P is capable of promoting the 
96

97

differentiation of MSCs in vitro (He et al. 2010). This suggests the possible synergistic mechanism of MSCs and S1P in the treatment of ALI/ARDS. On the one hand, while S1P plays a role in enhancing endothelial barrier function, MSCs modulates the host's immune response to injury through its proliferation, regeneration and anti-inflammatory effects. On the other hand, with the differentiation accelerating effect of S1P on MSCs, the MSCs have better immune regulation. The effect of MSC on the expression of S1P receptors and metabolic enzymes, leads to better in vivo homeostasis of S1P which in turn enhances the endothelial barrier.

Therefore in this study, by building an acute injury model of pulmonary endothelial cells induced by LPS, the regulatory effect of MSCs on the expression of S1P receptors and sphingosine kinase, in the treatment of acute lung injury was investigated. The efficacy of improving endothelial barrier function in acute lung injury when MSCs were used in combination with S1P and their possible synergistic mechanism are discussed. Thus it provides theoretical and experimental basis for the treatment of acute lung injury.

Materials and methods

Standards and reagents

Sphingosine-1-Phosphate (S1P) purchased from Sigma-Aldrich Company was dissolved in methanol and kept at $-20^{\circ} \mathrm{C}$. Lipopolysaccharide (LPS) E.coli O55:B5 purchased from SigmaAldrich Company was dissolved in saline and kept at $-20^{\circ} \mathrm{C}$.

\section{Antibody}

Anti-EDG-1 (S1PR1), Anti-EDG-5 (S1PR2) and Anti-EDG-3 (S1PR3) were purchased from Santa Cruz Company. Anti- $\beta$-actin was purchased from Sigma-Aldrich Company.

\section{Cell culture}

Newborn umbilical cord was taken under sterile conditions (The Affiliated Hospital of Military Medical Science Scientific Research Ethics Committee Approval Report, № ky-2015-3-17)and remnant blood in umbilical vein and artery was rinsed in D-HANKS solution containing $10^{5} \mathrm{U} / \mathrm{L}$ penicillin and $100 \mathrm{mg} / \mathrm{L}$ streptomycin. Collagenase IV solution was added to digest the cells, at $37^{\circ} \mathrm{C}$ for 2 hours. Single cell suspension of MSCs was obtained following filtration. After the 
123

124

125

126

127

128

129

130

\section{1}

132

133

134

135

136

137

138

139

140

141

142

143

144

145

146

147

148

cells were counted, the suspension was inoculated into $10 \mathrm{ml}$ MSC medium (Thermo Fisher, USA) in a $100 \mathrm{~mm}$ petri dish with a cell density of 2.5 to $4 \times 10^{4} / \mathrm{cm}^{2}$. After primary culture at $37^{\circ} \mathrm{C}$ and $5 \% \mathrm{CO}_{2}$ in an incubator for 7 to 9 days, first passage was carried out according to overall growth condition and local density. Incubation was continued until the density exceeds $80 \%$, repeated the above operations. Subculture generations at P3 to P5 were used for subsequent experiments. Human pulmonary artery endothelial cells (HPAECs) purchased from ScienCell Company were cultured in endothelial cell medium (ScienCell, USA) at $37^{\circ} \mathrm{C}$ in a $5 \% \mathrm{CO}_{2}$ in incubator. P5 to P8 cells were used for subsequent experiments.

\section{Acute Injury of cells}

HPAEC cells between P5 to P8 were inoculated into a 16-well E-Plate (ACEA Biosciences, USA) at a concentration of $2 \times 10^{4}$ per well in $100 \mu \mathrm{l}$, then cultured in an incubator at $37^{\circ} \mathrm{C}$ and $5 \%$ $\mathrm{CO}_{2}$ for 12 hours following which LPS was added at varying concentrations of $0,0.5,1,2$ and 5 $\mu \mathrm{M}$. The 16-well E-Plates were placed within the Real Time Cellular Analysis (RTCA) System (ACEA Biosciences, USA) and cultured in an incubator at $37^{\circ} \mathrm{C}$ and $5 \% \mathrm{CO}_{2}$. Micro-electrical impedance of HPAEC cells were detected in real-time to investigate the effects of acute injury caused by LPS on HPAEC cells, at different concentrations.

HPAEC cells between P5 to P8 were inoculated into a 24-well cell culture plate (Corning, USA) at a concentration of $1 \times 10^{5}$ per well in $600 \mu$, then cultured in incubator at $37^{\circ} \mathrm{C}$ and $5 \%$ $\mathrm{CO}_{2}$ for 6 to 12 hours after LPS was added at a final concentration of $1 \mu \mathrm{M}$. Cells were collected at different time points and the expression of TNF- $\alpha$ was detected to investigate the acute injury effects of LPS on HPAEC cells.

\section{Cell co-culture}

HPAEC cells between P5 to P8 were inoculated into a 16-well E-Plate (ACEA Biosciences, USA) in $100 \mu \mathrm{l}$ at a concentration of $2 \times 10^{4}$ per well and then LPS at a concentration of $1 \mu \mathrm{M}$ was added. In the meantime, $60 \mu \mathrm{l}$ of MSCs at different proportions (wherein the ratio of HPAEC and MSC were1:1, 1:2, and 1:4, the control group is MSCs medium only) were inoculated into a 16- 
149

150

151

152

153

154

155

156

157

158

159

160

161

162

163

164

165

166

167

168

169

170

171

172

173

174

well E-Plate Insert (ACEA Biosciences, USA), Then Insert was placed into Receiver Plate containing $100 \mu \mathrm{l}$ of MSC medium. The 16-well E-Plates were placed within the RTCA System, together with the Receiver Plates, were cultured in an incubator at $37^{\circ} \mathrm{C}$ and $5 \% \mathrm{CO}_{2}$ for 12 hours. Following that, medium in E-Plates was discarded and replaced with $100 \mu \mathrm{l}$ of fresh endothelial cell medium and the Insert was filled to $60 \mu \mathrm{l}$ with MSC medium in the corresponding Receiver Plates. Then Inserts was placed into E-Plates containing HPAECs and co-cultured within the RTCA System at $37^{\circ} \mathrm{C}$ and $5 \% \mathrm{CO}_{2}$ for $8-24$ hours. Micro-electrical impedance of HPAEC cells were detected in real-time to investigate the effect of MSCs on HPAEC cell injury.

HPAEC cells between P5 to P8 in $600 \mu 1$ medium were inoculated into a 24-well cell culture plate at a concentration of $1 \times 10^{5}$ per well following which LPS at a concentration of $1 \mu \mathrm{M}$ was added. Meanwhile, $100 \mu \mathrm{l}$ of MSCs with different proportions (wherein the ratio of HPAEC and MSC were 1:1,1:2, and 1:4, the control group is MSCs medium only) were inoculated into the upper compartment of a 24 well Transwell plate (0.4 $\mu \mathrm{m}$ Polyester Membrane, Corning, USA), and $600 \mu \mathrm{l}$ of MSC medium was added into the lower compartment. The 24 -well cell culture plates and Transwell plates were incubated at $37^{\circ} \mathrm{C}$ and $5 \% \mathrm{CO}_{2}$ for 12 hours. Then the medium in the cell culture plates was discarded and replaced with $600 \mu \mathrm{l}$ of fresh endothelial cell medium, the upper compartment of the Transwell plates was filled to $100 \mu \mathrm{l}$ with MSC medium in the corresponding lower compartments. After 8 to 24 hours of co-culture with the upper compartment of Transwell plates placed into cell culture plates, HPAEC cells were collected. The effect of MSCs on HPAEC cell injury was investigated by determining the expression change of TNF- $\alpha$.

Detection of Cell Micro-electronics impedance

16-well E-Plate was placed on Xcelligence RTCA DP system (ACEA Biosciences, USA) and incubated at $37^{\circ} \mathrm{C}$ and $5 \% \mathrm{CO}_{2}$. Detection was determined according to the manufacturer's instructions.

\section{Real-time quantitative PCR}

Total RNA was extracted from collected HPAEC cells using RNeasy®Mini Kit (Qiagen, 
175 USA) according to manufacturer's instructions. With $1 \mu \mathrm{g}$ of RNA as template, reverse 176 transcription reaction was performed using QuantScript RT Kit (TIANGEN, Beijing, China) 177 according to manufacturer's instructions. Utilizing Bio-Rad iQ ${ }^{\mathrm{TM}} 5$ Multicolor Real-Time PCR 178 Detection System and with GAPDH as internal reference, fluorescence intensity was detected

179 180 using SuperReal PreMix SYBR Green (TIANGEN, Beijing, China) according to the manufacturer's instructions. The primers used are shown in Table 1. Relative expression level of mRNA was calculated by $2-\Delta \Delta \mathrm{Ct}$ method.

\section{Western blot analysis}

The collected HPAEC cells were washed with PBS twice. Then $200 \mu 1$ lysis buffer was added, which contains 20mM Tris- $\mathrm{HCl}$ (pH 7.5), 150 mM NaCl, 1 mM EDTA, 1 mM EGTA, 1\% Triton $\mathrm{X}-100,2.5 \mathrm{mM}$ pyrophosphate sodium phosphate, $1 \mu \mathrm{g} / \mathrm{ml}$ leupeptin, $1 \mu \mathrm{g} / \mathrm{ml}$ aprotinin and protease inhibitors (Roche, USA). Cell lysate was centrifuged at $10,000 \times g$, room temperature for $10 \mathrm{~min}$ and the supernatant was collected. After protein concentration was measured using BCA method, 20 to $50 \mu \mathrm{g}$ of protein was separated by $12 \%$ SDS-PAGE, then transferred to a PVDF membrane and sealed with TBST buffer containing 5\% BSA. Specific antibodies were added and incubated overnight. On the next day, after washing and secondary antibody incubation, chromogenic assay was performed using ECL chemiluminescence kit (GE Healthcare, USA).

\section{Statistical analysis}

All experimental data are represented as mean \pm standard deviation (mean $\pm \mathrm{SD}$ ). One-way ANOVA (Dunnett test) was adopted in multiple group comparison. Data was analyzed with software GraphPad Prism 6.0. $p<0.05$ indicates that the difference is statistically significant.

\section{Results}

\section{Acute injury can be caused 12 hours after exposure of HPAECs to $1 \mu \mathrm{M}$ LPS}

LPS is widely used in the preparation of acute lung injury model. However, there is no standard procedure that has been developed. Literatures shows that acute injury induced by LPS will cause significant endothelial cell barrier disorder and increase in permeability. In the meantime, there is abnormal expression of a variety of growth and inflammatory factors. 
202

203

204

205

206

207

208

209

210

211

212

213

214

215

216

217

218

219

220

221

222

223

224

225

226

227

228

229

230

Particularly, the expression of TNFa is significantly increased (Meduri et al. 2009). Here HPAECs were stimulated using LPS at varying final concentrations of $0,0.5,1,2$ and $5 \mu \mathrm{M}$ for 12 hours, respectively. The micro-electrical impedance changes of endothelial cells were detected by Real Time Cellular Analysis (RTCA) to reflect the changes in endothelial barrier function (Fig. 1A). Results show that compared to other concentrations, micro-electrical impedance dropped significantly when $1 \mu \mathrm{M}$ LPS was added to HPAECs (Fig. 1B) .This indicates that $1 \mu \mathrm{M}$ LPS is more effective in acute injury. Furthermore, HPAECs were stimulated by LPS at a concentration of $1 \mu \mathrm{M}$ for different time to detect the change in TNF- $\alpha$ expression. Results demonstrated that the expression of $\mathrm{TNF} \alpha$ was significantly increased at 12 hours after stimulation (Fig. 1C). This suggests that HPAECs stimulated by $1 \mu \mathrm{M}$ LPS for 12 hours can produce an acute cell injury model for subsequent studies.

Fig. 1 Real Time Cellular Analysis (RTCA) of micro-electrical impedance changes of HPAECs. A. A schematic showing the experimental design. B. Micro-electrical impedance of HPAECs detected in real-time after 12 hours of culture with different final concentrations of LPS. C. Histogram showing the change in TNF- $\alpha$ expression in HPAECs, at different time points using RT-PCR, in the presence of $1 \mu \mathrm{M}$ LPS $(* p<0.05)$.

\section{The effect of MSCs on acute injury of HPAECs}

HPAECs were incubated in a 24 -well plate with $1 \mu \mathrm{M}$ of LPS and were divided into 4 groups, based on the treatment timeline, control group, 8, 16 and $24 \mathrm{~h}$ treatment groups. Following this the cells were non-contact co-cultured with MSCs. The cells were collected and the change in expression of TNF $\alpha$ was examined by RT-PCR. Results show that the effective treatment window of acute endothelial cell injury by MSCs is $8 \mathrm{~h}$ (Fig. 2A).

In addition the treatment groups were divided into another 4 groups based on the cell ratio as, control group (HPAECs only), groups with inoculation ratio of HPAECs and MSCs 1:1, 1:2 and 1:4. They were non-contact co-cultured with HPAECs following treatment with LPS. Cells of each group were collected and the change in expression of TNF $\alpha$ was examined by RT-PCR. Results show that larger ratio of MSCs is not necessarily better. Best treatment effect was achieved when 
231

232

233

234

235

236

237

238

239

240

241

242

243

244

245

246

247

248

249

250

251

252

253

254

255

256

257

258

259

260

the ratio of HPAECs and MSCs was 1:2 (Fig. 2B). Results from label-free Real Time Cellular Analysis (RTCA) also show that, compared to that of the control group, the micro-electrical impedance of groups where HPAECs were co-cultured with MSCs were significantly higher and reached their peak after 10 hours. Among them, micro-electrical impedance of the group with the ratio of HPAECs to MSCs 1:2 was the highest. 15 hours later, micro-electrical impedance of the control group was significantly higher than that of the other groups (Fig. 2C). The results were consistent with the change in expression of TNF- $\alpha$, indicating that the effect of MSCs on acute injury of HPAECs was best at a ratio of 1:2 and the best treatment time is about 8 to 10 hours.

Fig. 2. A. The change in expression of TNF $\alpha$ at different time points when injured HPAECs were co-cultured with MSCs. Histogram showing TNF $\alpha$ expression in HPAECs, using RT-PCR. $\left({ }^{*} p<0.05, * * p<0.01\right)$. B. The change in expression of TNF $\alpha$ when injured HPAECs were cocultured with different proportions of MSCs as examined at different time points by RT-PCR $\left({ }^{*} p<0.05\right.$ and $\left.{ }^{*} p<0.01\right)$. C. The micro-electrical impedance of HPAECs exposed to LPS and cocultured with MSCs as examined using RTCA system.

\section{The effect of S1P acute HPAEC injury}

Sphingosine-1-phosphate (S1P) is a biologically active ubiquitous sphingomyelin. It is mainly produced by platelets and also present in plasma and tissues. When its physiological concentration is in the range of 0.2 to $1.1 \mu \mathrm{M}$, it can effectively enhance the endothelial barrier function. In this study, a HPAEC acute injury model was established using $1 \mu \mathrm{M}$ LPS and treated with S1P at final concentrations of $0,0.5,1,2$, and $5 \mu \mathrm{M}$. The effect of LPS on HPAEC injury was studied by RTCA experiment. Results show that, S1P within physiological concentrations can effectively enhance the micro-electrical impedance of HPAECs (Fig. 3A). In addition HPAECs were cultured in a 16-well E-Plate and $1 \mu \mathrm{M}$ LPS was added to induce injury. Meanwhile, MSCs at the twice concentration was inoculated into a 16-well E-Plate Insert. After incubated for 12 hours, the LPS medium was discarded and S1P at concentrations of $0,0.5,1,2$, and $5 \mu \mathrm{M}$ were added in the E-Plate and E-Plate Insert for co-culture, then the effect on HPAEC injury was studied by RTCA. Results show that S1P within physiological concentration $(0.5$ and $1 \mu \mathrm{M})$ can effectively enhance the micro-electrical impedance of HPAECs and alleviate LPS induced injury to HPAECs 
261 (Fig. 3B). Both sets of data show that the enhancing effect of $0.5 \mu \mathrm{M}$ S1P on micro-electrical

262

263

264

265

266

267

268

269

270

271

272

273

274

275

276

277

278

279

280

281

282

283

284

285

286

287 288

impedance was superior to that of $1 \mu \mathrm{M}$ S1P in the presence or absence of MSCs. In addition, using a Transwell plate, HPAECs exposed to $1 \mu \mathrm{M}$ LPS for 12 hours were co-cultured with MSCs at a ratio of 1:2 and $0.5 \mu \mathrm{M} \mathrm{S1P}$ for 8 hours. HPAECs were collected and the change in expression of TNF- $\alpha$ was determined. Results show that compared to MSCs alone, the down-regulation of TNF$\alpha$ expression was more significant when S1P was combined with MSCs (Fig. 3C).

Fig. 3. The combined effect of LPS and S1P on micro-electrical impedance of HPAECs. A. The effect of S1P on HPAEC injury studied using RTCA. Results show that, S1P within physiological concentration $(0.5$ and $1 \mu \mathrm{M})$ can effectively enhance the micro-electrical impedance of HPAECs. B. The combined effect of MSCs and S1P on micro-electrical impedance of LPS injuried HPAECs as examined using the RTCA system. C. Change in expression of TNF $\alpha$ in injured HPAECs when treated with S1P and MSCs using RT-PCR $(* * p<0.01)$.

\section{Effects of S1P and MSCs combination therapy compared to MSCs alone}

In order to further investigate the possible synergistic mechanism of MSC and S1P in the treatment of HPAEC injury, the change in S1P receptors and sphingosine kinase expression in HPAECs treated with MSCs alone or the combination of MSCs with S1P, were determined using RT-PCR. After 12 hours following exposure to LPS, the expression changes of S1P receptors (S1PR1, S1PR2 and S1PR3) and sphingosine kinases (SphK1 and SphK2) in HPAECs were studied using RT-PCR. Results demonstrate that the expression of target genes were dramatically increased following LPS induced injury. The expression of S1PR1 and S1PR3 were increased significantly, while that of S1P2 was not. There was no significant difference in the expression levels of SphK1 and SphK2 following LPS induced injury (Fig. 4). These results indicate that the S1P related genes could act as potential therapeutic targets. It also suggests that acute lung injury is a complex pathophysiological process and the treatment directed to a single target may not achieve the best efficacy. The combination of MSCs and S1P may become an effective therapy. 
289 Fig. 4. S1P receptor and Sphingosine kinase expression following acute injury. A.

290 Representative figure showing expression change of S1P receptors 1, 2 and 3. B. Histogram

291 demonstrating the expression change of sphingosine kinases 1 and 2 . $(* p<0.05, * * p<0.01)$.

292

293

294

295

296

297

298

299

300

301

302

303

304

305

306

307

308

309

310

311

312

313

314

315

316

317

318 2

Furthermore, after 12 hours of LPS exposure, the medium was changed. According to the coculture condition, HPAECs were divided into 3 groups, that is, control group, MSC therapy group and combined therapy group (with $0.5 \mu \mathrm{M} \mathrm{S1P}$ and MSCs at a ratio of 1:2 of HPAECs). After 8 hours of non-contact co-culture, HPAECs cells were collected and total RNA were extracted. The changes in expression of S1P related genes were detected using RT-PCR. Results show that compared with MSC alone, the down-regulation of TNF- $\alpha$ expression was significant when MSCs were used in combination with S1P. This indicated that there is a possible synergistic mechanism, between MSCs and S1P, in the treatment of acute injury (Fig. 5A). The regulation results of S1P receptors show that when MSCs work on injured HPAECs, its regulation of S1P receptors were different, affecting only S1PR2 and S1PR3. The expression of S1PR2 and S1PR3 were further reduced when MSCs were used in combination with S1P. There was no significant difference regulatory effect on S1PR1 irrespective of whether MSCs were used alone or in combination with S1P (Fig. 5B).

Fig. 5. Effect of MSC and S1P combination therapy on TNF- $\alpha$, S1P receptors and Sphingosine kinases. A. Histogram demonstrating the expression change of TNF- $\alpha$. B. Histogram demonstrating the expression change of S1P receptors 1, 2 and 3. C. Histogram demonstrating the expression change of sphingosine kinases 1 and $2(* * p<0.01)$.

Studies have shown that the intracellular level of S1P is tightly regulated by the balance between synthesis and degradation. In vivo, sphingomyelin (SM) is catalyzed to produce ceramide, which is further hydrolyzed to sphingosine. Sphingosine is able to produce S1P by phosphorylation catalyzed by sphingosine kinase (SphK1 and SphK2). Our study results demonstrate that the expression of sphingosine kinase (SphK1 and SphK2) differs in HPAECs following LPS injury. When MSCs were used alone, it mainly worked on SphK1, the expression level of which was significantly reduced. When MSCs were used in combination with S1P, the expression of SphK2 
319

320

321

322

323

324

325

326

327

328

329

330

331

332

333

334

335

336

337

338

339

340

341

342

343

344

345

346

347

was also significantly reduced (Fig. 5C). Through analyzing the expressions of S1P related genes, we found that when MSCs were combined with S1P, their effect was not simply the superimposition of each other, but more significant. This suggests that there might be some synergistic mechanism between MSCs and S1P in the treatment of acute injury.

\section{Impact of MSC and S1P on S1P receptor Expression in acute injury}

After 12 hours of HPAECs exposed to LPS were divided into 3 groups, based on co-culture conditions as, control group, MSC therapy group and combined therapy group $(0.5 \mu \mathrm{M} \mathrm{S1P}$ and MSCs at a ratio of 1:2 to HPAECs). After 8 hours of non-contact co-culture, HPAECs cells were collected and total proteins were extracted. The changes in expression of S1P receptors were detected through Western-Blot. The results were consistent with that from the RT-PCR. The results of regulation of different S1P receptors were different when MSCs were added, where S1PR2 and S1PR3 were the main targets. The effect on S1PR1 was not significant whether MSCs were used alone or in combination with S1P (Fig. 6).

Fig. 6. S1P receptors expression in HPAECs treated with MSCs. Pictograph showing Western blot analysis of S1P receptor expression HPAECs exposed to LPS that were treated with MSCs alone or in combination with S1P.

\section{Discussion}

Sphingosine-1-phosphate (S1P) is an agonist mainly present in plasma and tissues. It is an important regulator of vascular endothelial cell permeability and fluid balance in vivo and is able to enhance endothelial barrier. Its homeostasis in vivo is tightly regulated by the balance between synthesis and degradation. In vivo, sphingomyelin (SM) is catalyzed by Sphingomyelinase to produce ceramide (Cer), which produces sphingosine in hydrolysis catalyzed by Neuraminidase. Phosphorylation of sphingosine produces S1P catalyzed by sphingosine kinase (SphK1 and SphK2). Physiological concentrations of S1P plays an important role in maintaining endothelial barrier function. It has been demonstrated that the activity of SphK1 and the dynamic concentration balance of SphK1/S1P axis, are necessary for the modulation of inflammatory signals and are related to the regulation of innate, adaptive and other immune cells. Studies have also shown that 
348 abnormal changes in SphK1 or S1P lead to many inflammatory and autoimmune diseases, 349 including asthma, rheumatoid arthritis, sepsis, inflammatory bowel disease and so on(Spiegel \& 350 Milstien 2011). Our study found that in LPS induced acute injury of HPAECs, the expression of 351 SphKs was increased dramatically. When MSCs were used alone, only the expression of SphK1

352

353

354

355

356

357

358

359

360

361

362

363

364

365

366

367

368

369

370

371

372

373

374 was reduced, and there was no significant impact on the expression of SphK2. When MSCs were used in combination with S1P, the expression of SphK1 and SphK2 were reduced simultaneously.

This shows that MSCs play a role in the treatment of acute lung injury by regulating the expression and activity of SphKs. It also suggests that a possible synergistic mechanism may exist, between MSCs and S1P, in the treatment of acute HPAEC injury.

Studies on S1P show that endothelial barrier enhancement mediated by S1P is completed by activating the Gi and Racl signaling pathway through the S1P receptor(Yu et al. 2012). There are five different types of S1P receptors (S1PR1-5) and their distribution and function are different. Those in endothelial cells are mainly S1PR1-3. Wherein the S1P receptor 1 (S1PR1) has a more important biological role in enhancing endothelial barrier, the S1PR2 suppresses the endothelial barrier function (Blaho \& Hla 2014). Studies have shown that when the selective S1PR1 competitive antagonist is used in experimental mice, pulmonary endothelial cells integrity is compromised (Rosen et al. 2007; Sanna et al. 2006). Besides, it has also been found that stimulation of nitrification and release of S1PR3 in HPAECs helps to suppress the endothelial barrier function (Sun et al. 2012). These demonstrates that S1P receptors can be the therapeutic targets of ALI/ARDS to improve endothelial cell barrier. In this study We found that when MSCs works on acute injured HPAECs, the regulation of individual S1P receptors were different, which was mainly acted on S1PR2 and S1PR3. The expression of S1PR2 and S1PR3 were further reduced when MSCs were used in combination with S1P. The regulatory effect on S1PR1 was not significant irrespective of whether MSCs were used alone or in combination with S1P. This indicates that MSCs may play a role in enhancing endothelial barrier function by reducing the expression of S1PR2 and S1PR3 and enhancing specificity of S1P receptors.

Clinical studies have shown that any single target and drug are not likely to reverse the severe 
375

376

377

378

379

380

381

382

383

384

385

386

387

388

389

390

391

392

393

394

395

396

397

398

399

400

401

pathological injury caused by ALI/ARDS and achieve great therapeutic effect quickly. Innovative multi-target synergistic treatment mechanism may be an effective method to solve this problem. In essence, stem cell therapy does not work on a single target or as a single drug in therapy, but on multiple targets based on biological distress signals from the diseased tissues and as multiple therapies. Our study found that when MSCs worked on HPEACs with acute injury, the expressions of S1P receptors and sphingosine kinase were regulated. This indicates that MSCs are able to affect the expression of multiple S1P related genes, improve endothelial barrier and cure acute lung injury. Meanwhile, as an endogenous bioactive molecule, S1P is an important regulator of vascular endothelial cell permeability and fluid balance. In the treatment of acute lung injury, it plays an important role in improving endothelial barrier by regulating the expression of key enzymes in anabolic processes and specific receptors on signaling pathways. Our study results demonstrate that both MSCs and S1P can alleviate acute lung injury. But the combined use of MSCs and S1P shows significant efficacy in the regulation of S1P related gene expression, suggesting that some synergism between MSCs and S1P may exist in the treatment of acute injury. Further studies are need to determine whether MSCs when used in combination with S1P, further improve the endothelial barrier and if a possible synergistic mechanism exists and maybe to provide a novel therapeutic strategy in the treatment of acute lung injury.

\section{Conclusions}

In this study we built a pulmonary endothelial cell model of acute injury by LPS, and investigated the regulation of S1P receptors and sphingosine kinases expression by MSCs combined with S1P. The study results demonstrated that both MSCs and S1P can alleviate acute lung injury. When MSCs were used alone, only the expression of SphK1 was reduced, and there was no significant impact on the expression of SphK2. When MSCs were used in combination with S1P, the expression of SphK1 and SphK2 were reduced simultaneously. We also found that when MSCs works on acute injured HPAECs, the regulation of individual S1P receptors were different, which was mainly acted on S1PR2 and S1PR3. The expression of S1PR2 and S1PR3 
402

403

404

405

406

407

408

409

410

411

412

413

414

415

416

417

418

419

420

421

422

423

424

425

426

427

428

429

430

431

432

433

434

435

436

437

438

439

440

were further reduced when MSCs were used in combination with S1P. These results show that

MSCs play a role in the treatment of acute lung injury by regulating the expression and activity of

S1P related genes, when MSC combined with S1P there is a possible synergistic mechanism exists.

\section{References}

Blaho VA, and Hla T. 2014. An update on the biology of sphingosine 1-phosphate receptors. Journal of Lipid Research 55:1596-1608. DOI: 10.1194/jlr.R046300

Brower RG, Matthay MA, Morris A, Schoenfeld D, Thompson BT, and Wheeler A. 2000. Ventilation with lower tidal volumes as compared with traditional tidal volumes for acute lung injury and the acute respiratory distress syndrome. The Acute Respiratory Distress Syndrome Network. N Engl J Med 342:1301-1308.

Bruno S, Grange C, Deregibus MC, Calogero RA, Saviozzi S, Collino F, Morando L, Busca A, Falda M, and Bussolati B. 2009. Mesenchymal stem cell-derived microvesicles protect against acute tubular injury. Journal of the American Society of Nephrology 20:1053-1067. DOI: 10.1681/ASN.2008070798

Cepkova M, and Matthay MA. 2006. Pharmacotherapy of Acute Lung Injury and the Acute Respiratory Distress Syndrome. Journal of Intensive Care Medicine 21:119-143. DOI: $10.1177 / 0885066606287045$

Chang YS, Oh W, Choi SJ, Sung DK, Kim SY, Choi EY, Kang S, Jin HJ, Yang YS, and Park WS. 2009. Human umbilical cord blood-derived mesenchymal stem cells attenuate hyperoxiainduced lung injury in neonatal rats. Cell Transplantation 18:869-886. DOI: 10.3727/096368909X471189

Cuvillier O, Pirianov G, Kleuser B, Vanek PG, Coso OA, Gutkind JS, and Spiegel S. 1996. Suppression of ceramide-mediated programmed cell death by sphingosine-1-phosphate. Nature 381:800-803. DOI: 10.1038/381800a0

Harting MT, Jimenez F, Xue H, Fischer UM, Baumgartner J, Dash PK, and Cox CS. 2009. Intravenous mesenchymal stem cell therapy for traumatic brain injury. Journal of Neurosurgery 110:1189-1197. DOI: 10.3171/2008.9.JNS08158

He X, H'Ng SC, Leong DT, Hutmacher DW, and Melendez AJ. 2010. Sphingosine-1-phosphate mediates proliferation maintaining the multipotency of human adult bone marrow and adipose tissue-derived stem cells. Journal of Molecular Cell Biology 2:199-208. DOI: 10.1093/jmcb/mjq011

Kumar P, Shen Q, Pivetti CD, Lee ES, Wu MH, and Yuan SY. 2008. Molecular mechanisms of endothelial hyperpermeability: implications in inflammation. Expert Reviews in Molecular Medicine 11:440-452. DOI: 10.1017/S1462399409001112

Lee MJ, Thangada S, Claffey KP, Ancellin N, Liu CH, Kluk M, Volpi M, Sha'Afi RI, and Hla T. 1999. Vascular Endothelial Cell Adherens Junction Assembly and Morphogenesis Induced by Sphingosine-1-Phosphate. Cell 99:301-312.

Müller-Redetzky HC, Suttorp N, and Witzenrath M. 2014. Dynamics of pulmonary endothelial 
barrier function in acute inflammation: mechanisms and therapeutic perspectives. Cell \& Tissue Research 355:657-673. DOI: 10.1007/s00441-014-1821-0

Meduri GU, Annane D, Chrousos GP, Marik PE, and Sinclair SE. 2009. Activation and Regulation of Systemic Inflammation in ARDS : Rationale for Prolonged Glucocorticoid Therapy. Chest 136:1631-1643. DOI: 10.1378/chest.08-2408

Moodley Y, Atienza D, Manuelpillai U, Samuel CS, Tchongue J, Ilancheran S, Boyd R, and Trounson A. 2009. Human Umbilical Cord Mesenchymal Stem Cells Reduce Fibrosis of Bleomycin-Induced Lung Injury. American Journal of Pathology 175:303-313. DOI: 10.2353/ajpath.2009.080629

Natarajan V, Dudek SM, Jacobson JR, Morenovinasco L, Huang LS, Abassi T, Mathew B, Zhao Y, Wang L, and Bittman R. 2013. Sphingosine-1-phosphate, FTY720, and sphingosine-1phosphate receptors in the pathobiology of acute lung injury. American Journal of Respiratory Cell \& Molecular Biology 49:6-17. DOI: 10.1165/rcmb.2012-0411TR.

Nemeth K, Leelahavanichkul A, Ps, Mayer B, Parmelee A, Doi K, Robey P, Leelahavanichkul K, Koller B, Brown J, and $\mathrm{Hu} \mathrm{X}$. 2008. Bone marrow stromal cells attenuate sepsis via prostaglandin $\mathrm{E}(2)$-dependent reprogramming of host macrophages to increase their interleukin-10 production. Nature Medicine 15:42-49. DOI: $\quad 10.1038 / \mathrm{nm} .1905$

Ortiz LA, Gambelli F, Mcbride C, Gaupp D, Baddoo M, Kaminski N, and Phinney DG. 2003. Mesenchymal stem cell engraftment in lung is enhanced in response to bleomycin exposure and ameliorates its fibrotic effects. Proceedings of the National Academy of Sciences of the United States of America 100:8407-8411. DOI: 10.1073/pnas.1432929100

Rosen H, Sanna MG, Cahalan SM, and Gonzalez-Cabrera PJ. 2007. Tipping the gatekeeper: S1P regulation of endothelial barrier function. Trends in Immunology 28:102-107. DOI: 10.1016/j.it.2007.01.007

Rubenfeld G, Caldwell E, Peabody E, Weaver J, Martin D, Neff M, Stern E, and Hudson L. 2005. Incidence and outcomes of acute lung injury. N Engl J Med 353:1685-1693.

Sanna MG, Wang SK, Gonzalezcabrera PJ, Don A, Marsolais D, Matheu MP, Wei SH, Parker I, Jo E, and Cheng WC. 2006. Enhancement of capillary leakage and restoration of lymphocyte egress by a chiral S1P1 antagonist in vivo. Nature Chemical Biology 2:434441. DOI: $10.1038 /$ nchembio804

Spiegel S, and Milstien S. 2011. The outs and the ins of sphingosine-1-phosphate in immunity. Nature Reviews Immunology 11:403-415. DOI: 10.1038/nri2974

Sun X, Singleton PA, Letsiou E, Zhao J, Belvitch P, Sammani S, Chiang ET, Morenovinasco L, Wade MS, and Zhou T. 2012. Sphingosine-1-phosphate receptor-3 is a novel biomarker in acute lung injury. American Journal of Respiratory Cell \& Molecular Biology 47:628-636. DOI: $10.1165 / \mathrm{rcmb} .2012-0048 \mathrm{OC}$

Valcz G, Krenács T, Sipos F, Leiszter K, Tóth K, Balogh Z, Csizmadia A, Müzes G, Molnár B, and Tulassay Z. 2011. The role of the bone marrow derived mesenchymal stem cells in colonic epithelial regeneration. Pathology Oncology Research Por 17:11-16. DOI: 10.1007/s12253-010-9262-x

Wang D, Zhang H, Liang J, Li X, Feng X, Wang H, Hua B, Liu B, Lu L, and Gilkeson GS. 2013. 
482

483

484

485

486

487

488

489

490

491

492

493

Allogeneic mesenchymal stem cell transplantation in severe and refractory systemic lupus erythematosus: 4 years of experience. Cell Transplantation 22:2267-2277. DOI: $10.3727 / 096368911 X 582769$

Wiedemann H, Wheeler A, Bernard G, Thompson B, Hayden D, deBoisblanc B, Connors A, Hite R, and Harabin A. 2006. Comparison of two fluid-management strategies in acute lung injury. N Engl J Med 354:2564-2575. DOI: 10.1056/NEJMoa062200

$\mathrm{Yu}$ H, Nishi T, and Kawahara A. 2012. The functional roles of S1P in immunity. Journal of Biochemistry 152:305-311. DOI: 10.1093/jb/mvs090

Zheng Z, Hu L, Ming S, † RX, Fu J, Lv J, Chen L, Lv S, Li Y, and Yu S. 2012. Human umbilical cord mesenchymal stem cells improve liver function and ascites in decompensated liver cirrhosis patients. Journal of Gastroenterology \& Hepatology 27:112-120. DOI: 10.1111/j.1440-1746.2011.07024.x 
Figure 1 (on next page)

Real Time Cellular Analysis (RTCA) of micro-electrical impedance changes of HPAECs. 
A
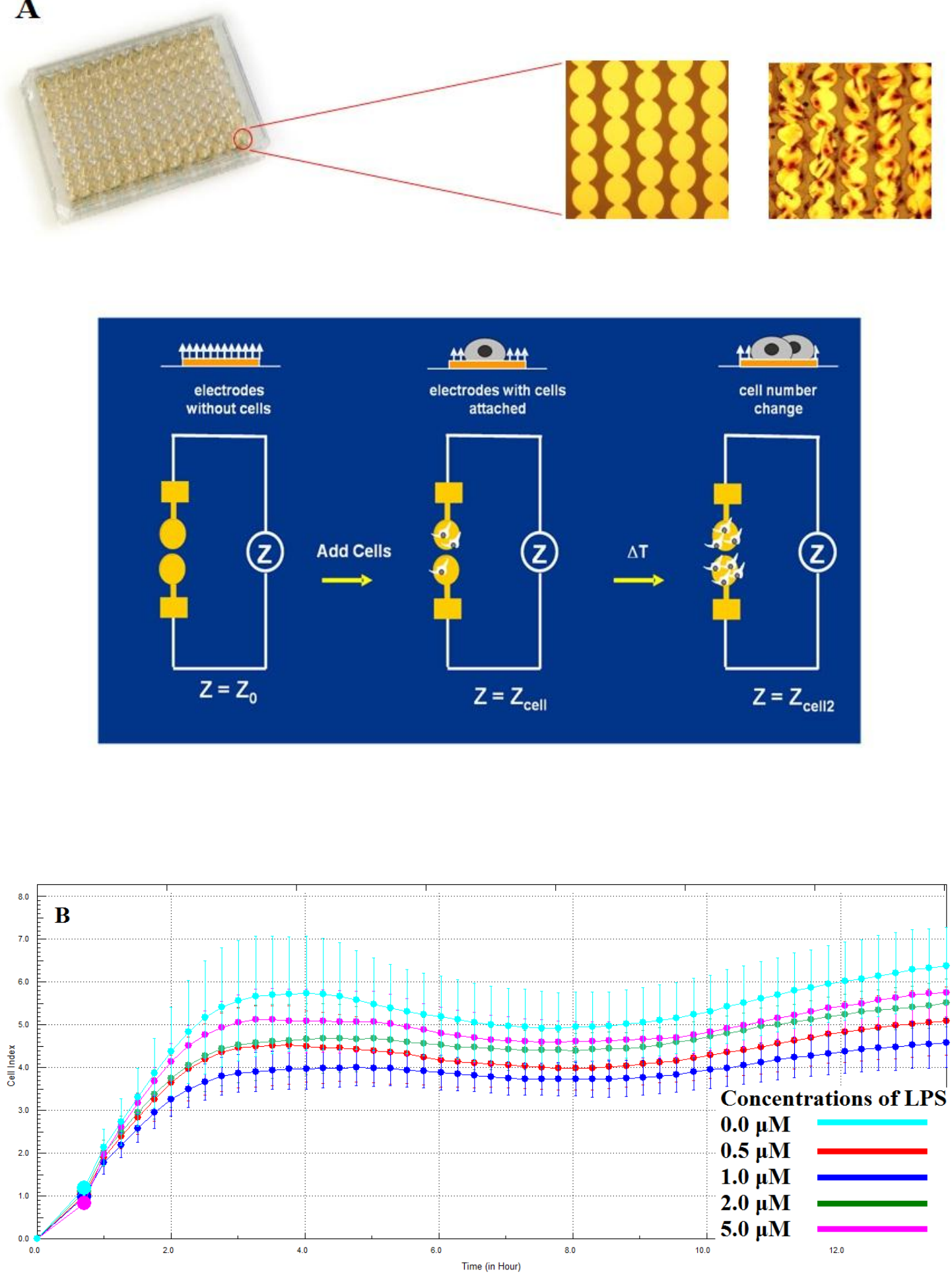


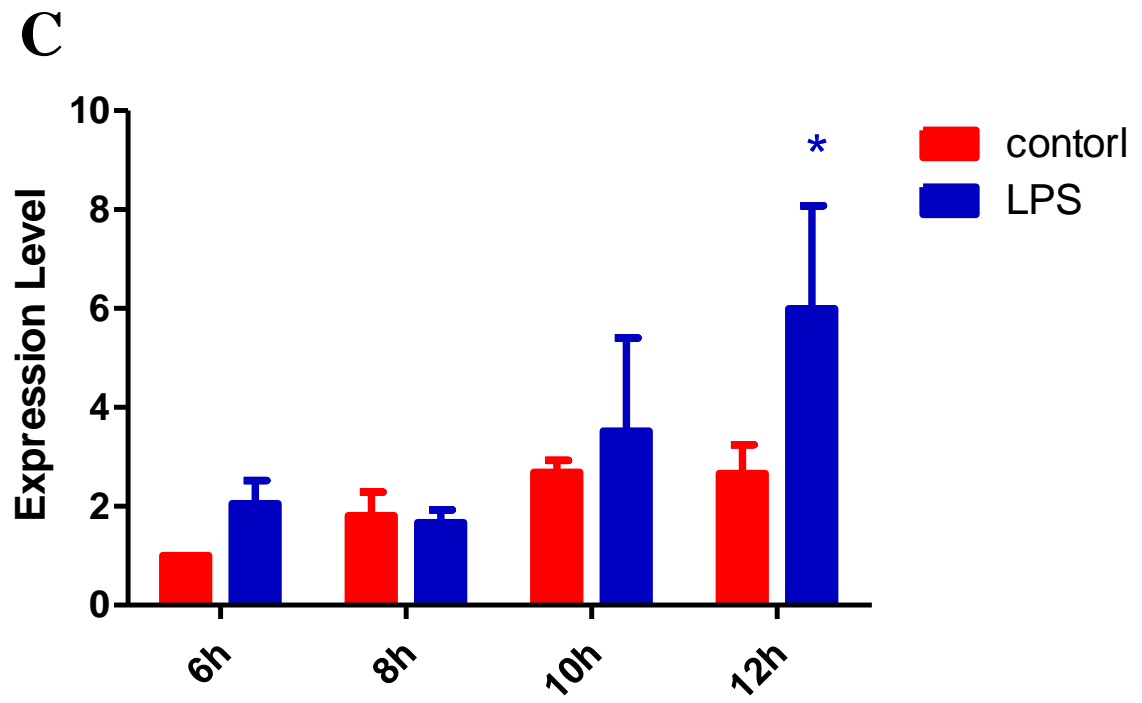




\section{Figure 2 (on next page)}

The change in expression of TNF $\alpha$ and micro-electrical impedance of HPAECs 

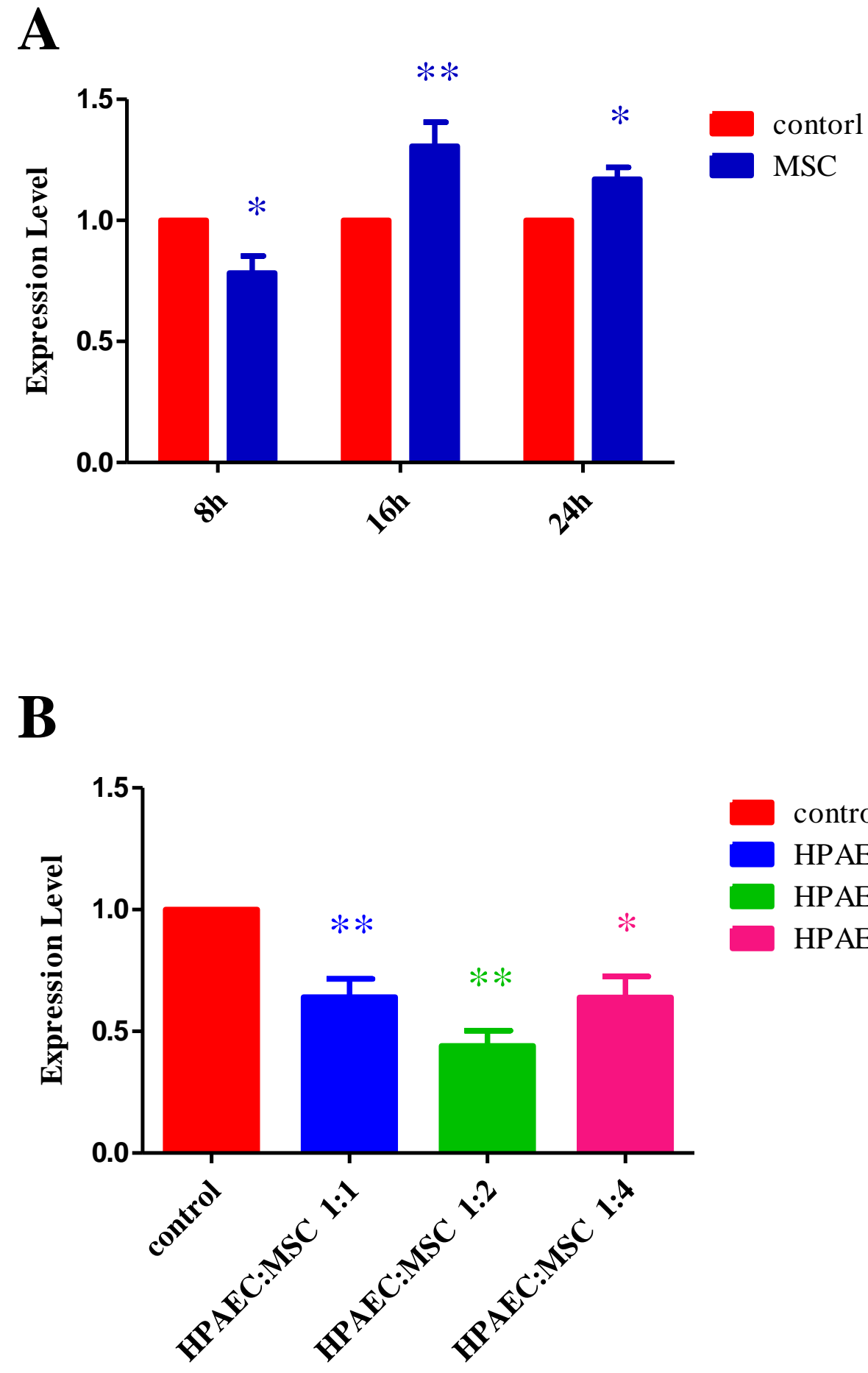


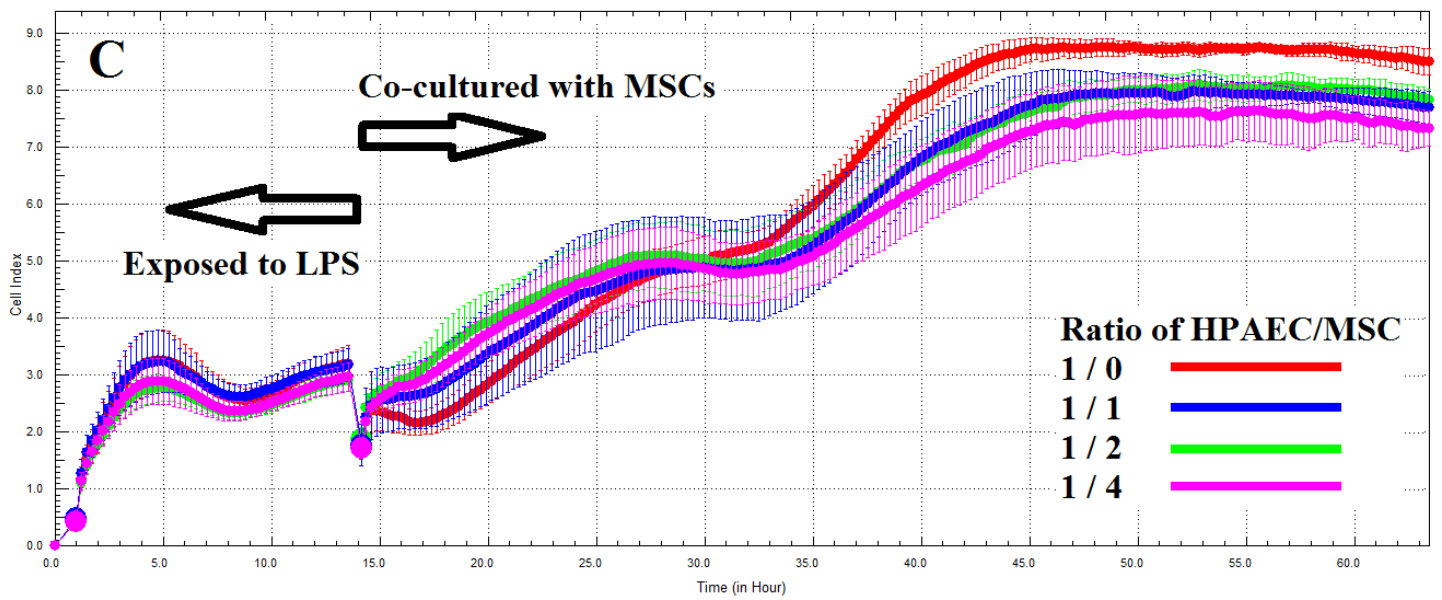


Figure 3 (on next page)

The combined effect of LPS and SIP on micro-electrical impedance of HPAECs. 

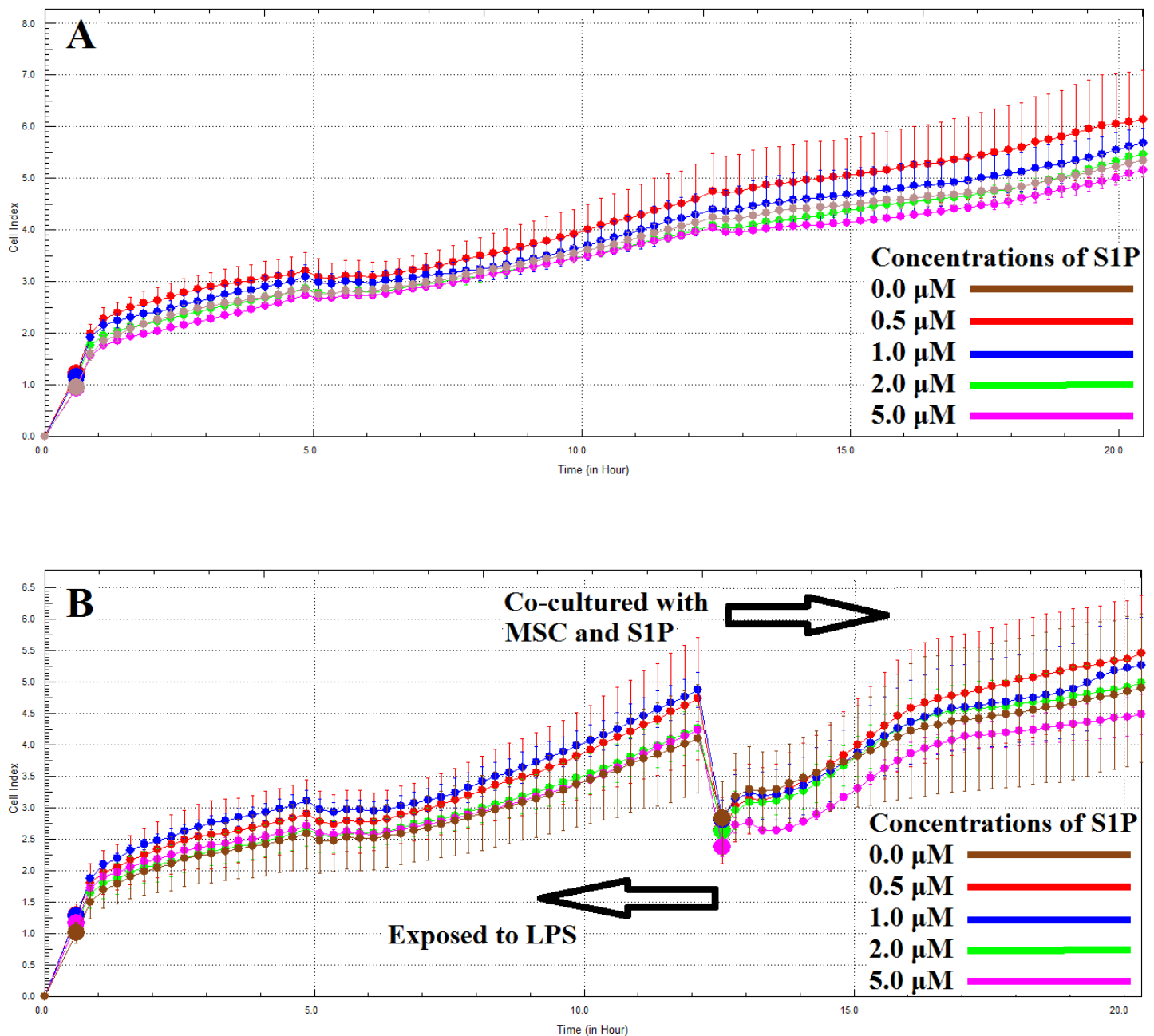

C

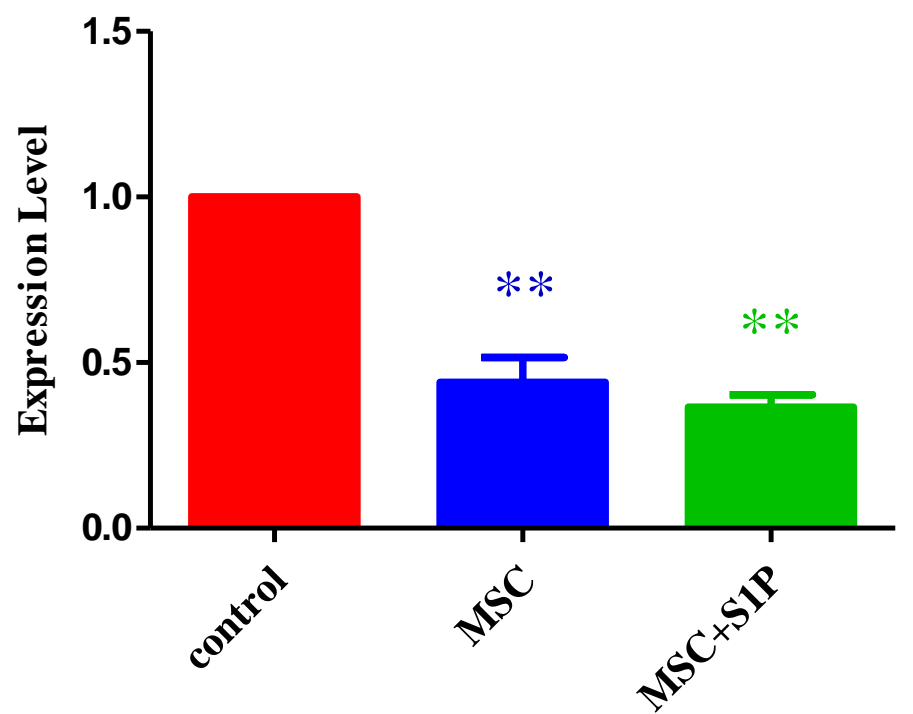

control

$\mathrm{MSC}$

$\mathrm{MSC}+\mathrm{S} 1 \mathrm{P}$ 
Figure 4 (on next page)

S1P receptor and Shpingosine kinase expression following acute injury. 
A

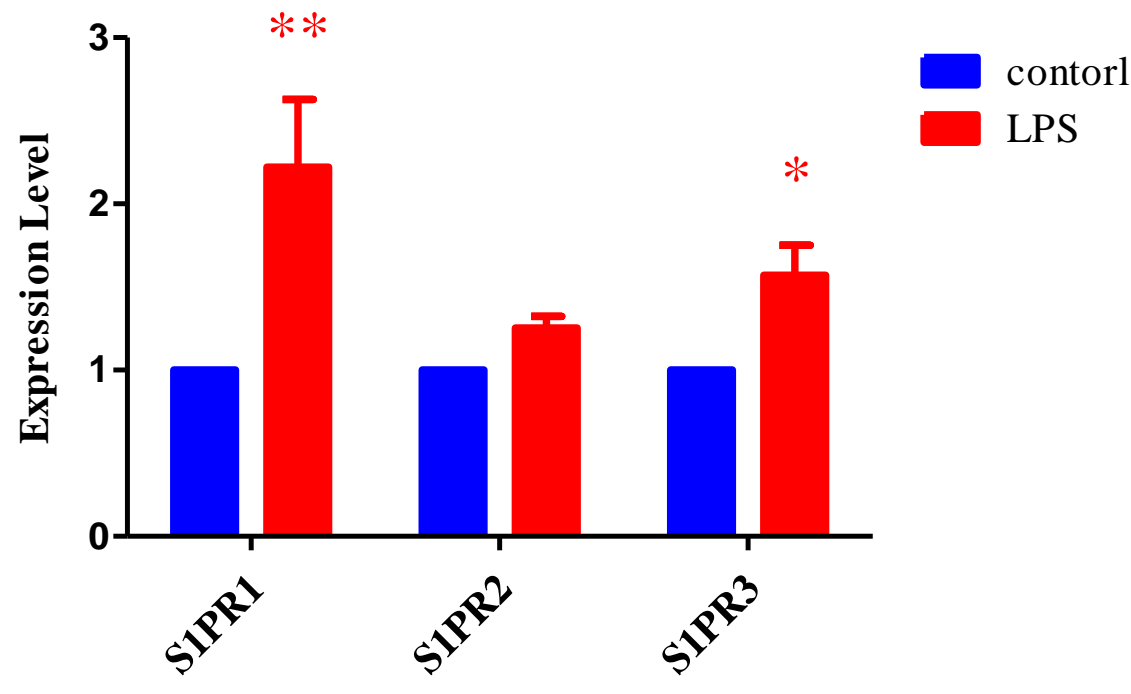

B

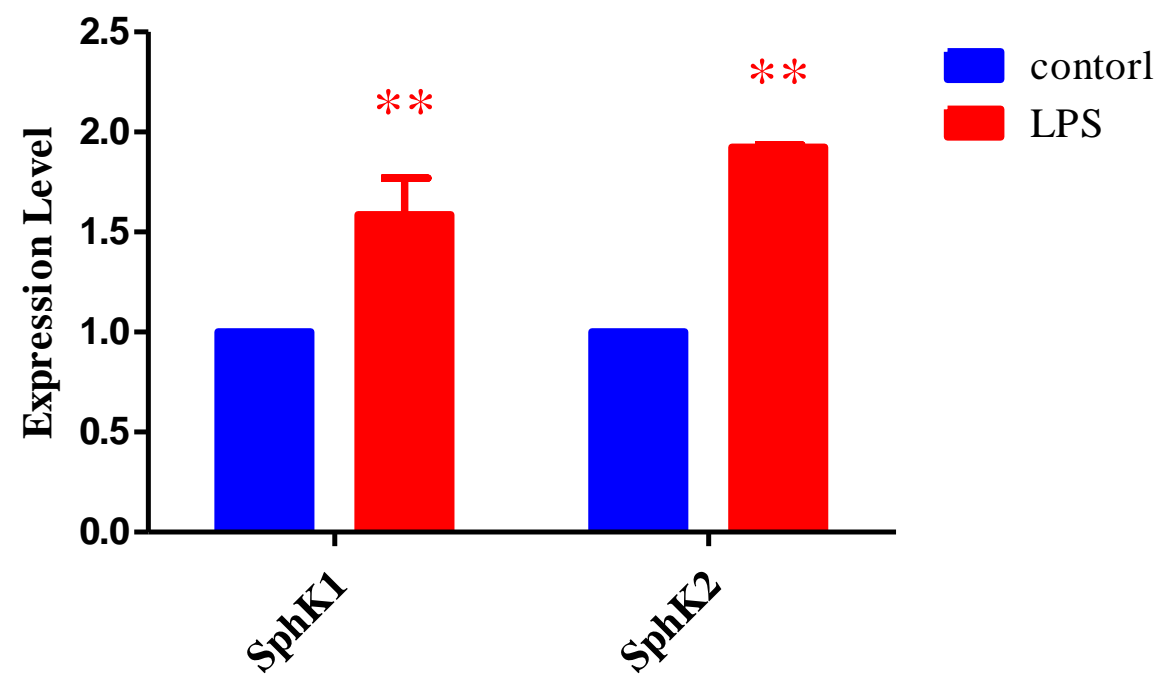


Figure 5 (on next page)

Effect of MSC and S1P combination therapy on TNF- a, S1P receptors and Sphigosine kinases. 
A

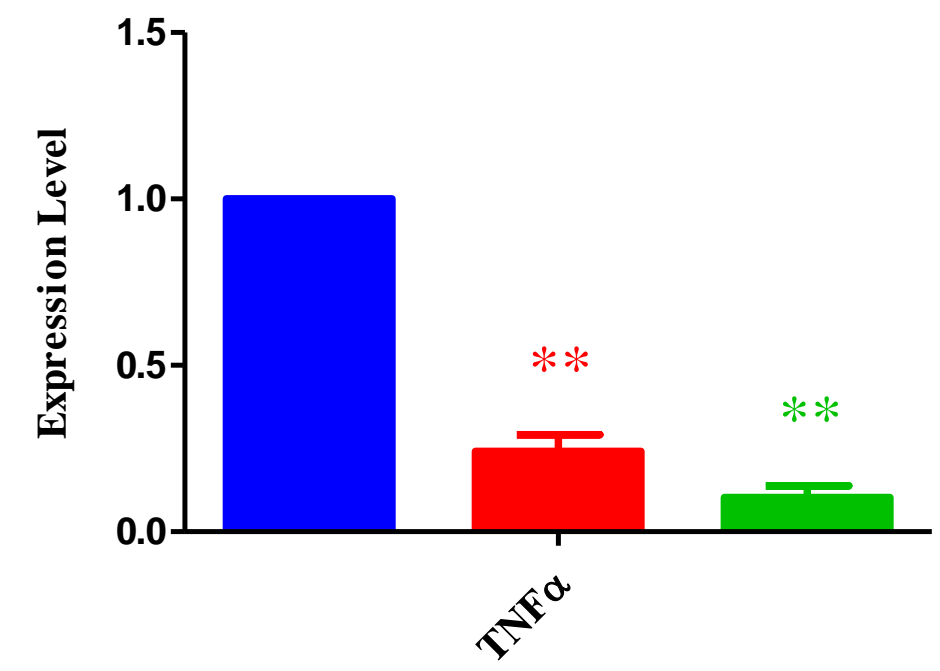

B

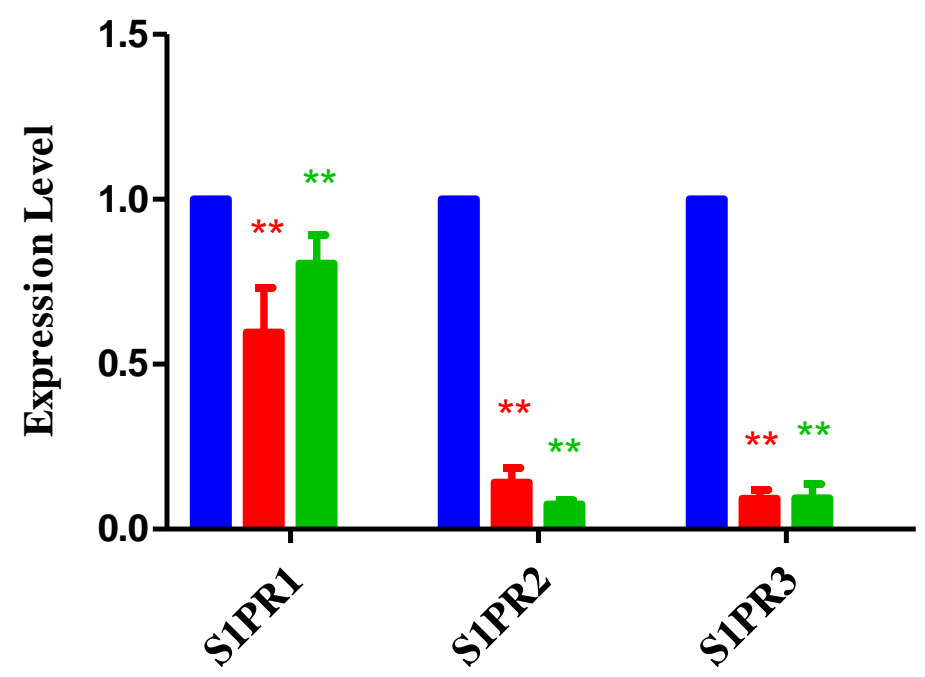




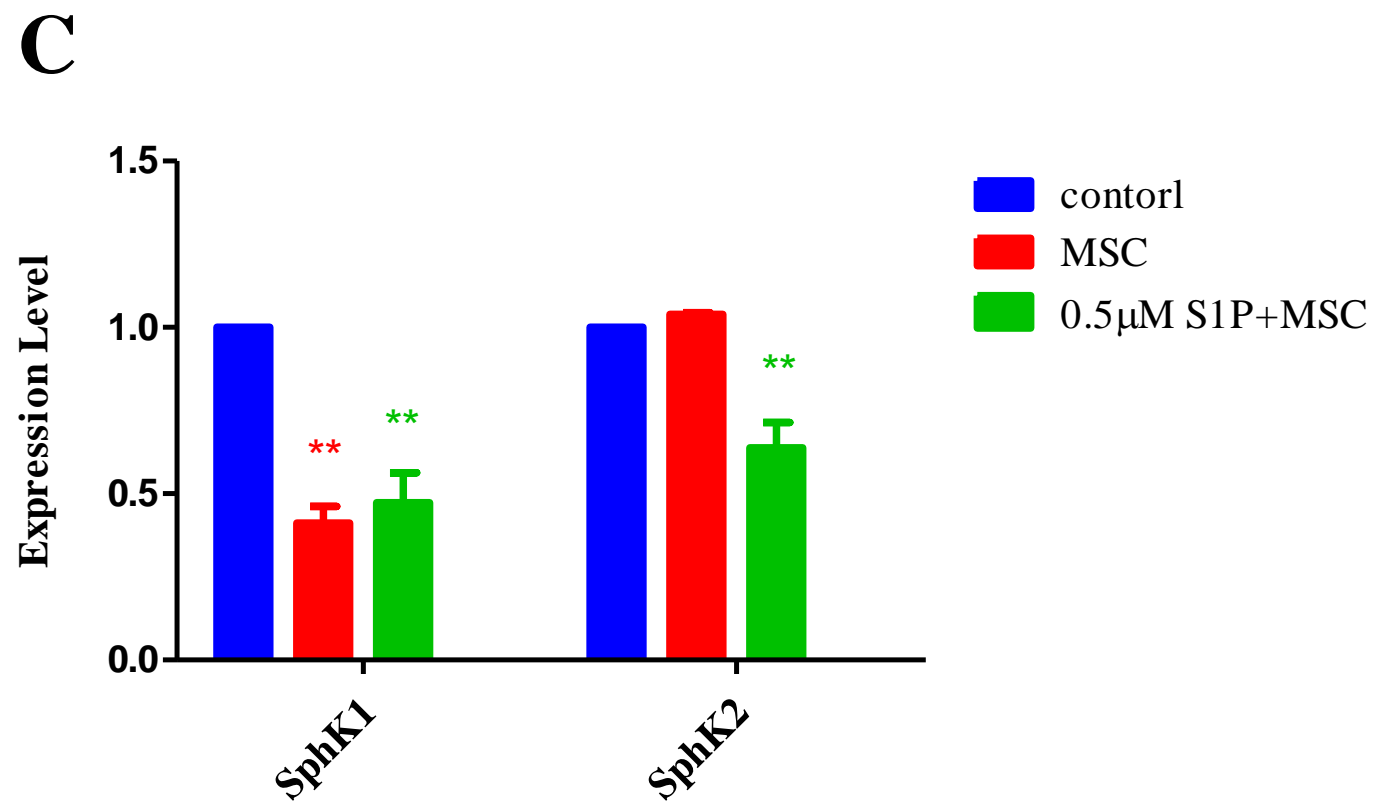


Figure $\mathbf{6}$ (on next page)

S1P receptor s expression in HPAECs treated with MSCs. 


\section{Peacontrol MSC}

S1PR3

S1PR2

S1PR1

$\beta$-Actin 
Table $\mathbf{1}$ (on next page)

Primer sequences 


\begin{tabular}{|c|c|}
\hline 引物 & 序列 \\
\hline S1PR1-F & 5'-GCACCAACCCCATCATTTAC-3' \\
\hline S1PR1-R & 5'-TTGTCCCCTTCGTCTTTCTG-3' \\
\hline S1PR2-F & 5'-CAAGTTCCACTCGGCAATGT-3' \\
\hline S1PR2-R & 5'-CAGGAGGCTGAAGACAGAGG-3' \\
\hline S1PR3-F & 5'-TCAGGGAGGGCAGTATGTTC-3' \\
\hline S1PR2-R & 5'-GAGTAGAGGGGCAGGATGGT-3' \\
\hline SphK1-F & 5'-TCTGGGCACCTTCCTGCGTC-3' \\
\hline SphK1-R & 5'-CTCACTGCCCAGGTGCGAGTG-3' \\
\hline SphK2-F & 5'-TGCTGGAAGGTGGGCGTC-3' \\
\hline SphK2-R & 5'-AATAGACTCCGCCCTCAGCC-3' \\
\hline TNF $\alpha-F$ & 5'-TGATCCCTGACATCTGGAATCTG-3' \\
\hline TNF $\alpha-R$ & 5'-GCCAAGGTCCACTTGTGTC-3' \\
\hline GAPDH & 5'-GAAGGTGAAGGTCGGAGTC-3' \\
\hline GAPDH & 5'-GAAGATGGTGATGGGATTTC-3' \\
\hline
\end{tabular}

